\title{
FUNCTIONS OF BOUNDED VARIATION AS SOLUTIONS OF DIFFERENTIAL SYSTEMS
}

\author{
F. W. STALLARD ${ }^{1}$
}

1. This paper investigates certain linear differential systems whose solutions are required to be merely functions of bounded variation. Subsequently the equivalence of such problems with appropriate integral equations with Lebesgue integrals and, also, their equivalence to certain integral equations with Stieltjes integrals is established. This study is a generalization of differential systems with interface conditions [6]. For such problems the usual condition that the solution be absolutely continuous is too stringent; in fact the interface conditions usually require a solution to have a jump discontinuity at each interface.

Matrix notation is used extensively. The elements of a matrix $A$ are denoted by $A_{i j}$. Any analytic properties such as continuity, bounded variation, or differentiability, postulated for a matrix are understood to be assumed for each element separately. The inverse of a matrix $A$, if it has one, is denoted by $A^{-1}$.

Let $\mathrm{G}_{n}(I)$ denote the class of all nonsingular $n \times n$ matrices $G$ which are absolutely continuous on the real interval $I$, and let $R_{n}(I)$ denote the class of all $n \times n$ matrices $R$ which have the following properties.

$$
R^{\prime}(x)=0,\left(^{\prime}\right)=\frac{d}{d x} \text {, a.e. on } I \text {. }
$$

$$
|\operatorname{det} R(x)| \geqq \delta>0 \text { for some } \delta \text { and for all } x \text { on } I \text {. }
$$

$$
R \text { is of bounded variation on } I \text {. }
$$

The following theorem plays a central role in all that follows.

THEOREM 1. Given an $n \times n$ matrix $F$ of bounded variation on an interval $I$; there exist matrices $G \in \mathcal{G}_{n}(I)$ and $R \in \mathcal{R}_{n}(I)$ such that

$$
F=R G+c E
$$

where $c$ is a suitably chosen constant and $E$ is the $n \times n$ identity matrix.

In fact once $c$ is chosen there is one and only one such representation for which $R(a)=E, a \in I$.

DEFinition. If $F$ is of bounded variation on $I$ and $R \in R_{n}(I)$, then $F$ is said to be of type $R$ on $I$ if and only if $R^{-1} F$ is absolutely continuous on $I$.

Received by the editors October 21, 1960 and, in revised form, May 8, 1961.

1 Supported by National Science Foundation Grant G-7343. 
The next result and its corollary are concerned with solutions $F$ of type $R$ for the system

$$
\begin{aligned}
Y^{\prime} & =P(x) Y, \\
Y(a) & =C .
\end{aligned}
$$

TheOREM 2. A necessary and sufficient condition that $(1.5,1.6)$ have a solution $F$ of type $R$ on an interval $I$ is that the system

$$
\begin{aligned}
& W^{\prime}=R^{-1}(x) P(x) R(x) W, \\
& W(a)=R^{-1}(a) C, \quad a \in I,
\end{aligned}
$$

have an absolutely continuous solution ${ }^{2} G$ on $I$, and in this case (1.5, 1.6) is equivalent to $(1.7,1.8)$ under the transformation $Y(x)=R(x) W(x)$.

If $R \in Q_{n}(I)$ so is $R^{-1}$; from this it follows that $R^{-1} P R$ is Lebesgue integrable if $P$ is Legesgue integrable. Using a well known theorem $[1$, p. 67] establishes the following.

Corollary. A sufficient condition for the existence and uniqueness of a solution $F$ of type $R$ of $(1.5,1.6)$ on $I$ is that $P$ be Lebesgue integrable on $I$.

It should be noted that Theorem 2 and its corollary hold if the solution matrices are $n \times k$.

The transformation $R$ can be used to duplicate for solutions of type $R$ much of the theory of absolutely continuous solutions of matrix differential equations. For example, by using the variation of constants formula for the absolutely continuous solutions of

$$
W^{\prime}=R^{-1} P R W+R^{-1} Q,
$$

and the Green's matrix $G(x, t)$ for the incompatible system consisting of (1.7) and

$$
[A R(a)] W(a)+[B R(b)] W(b)=0,
$$

one is led immediately to a corresponding variation of constants formula for solutions of type $R$ of

$$
Y^{\prime}=P Y+Q,
$$

and the Green's matrix $H(x, t)$ for the incompatible system (1.5) and

$$
A Y(a)+B Y(b)=0
$$

in particular, one has

${ }^{2}$ I.e. a solution in the "extended sense" [1, Chapter 2]. 


$$
H(x, t)=R(x) G(x, t) R^{-1}(t) .
$$

Suppose $P(x)$ is an $n \times n$ matrix which is Lebesgue integrable on $I=[a, b]$, and suppose for a positive integer $k$ there are given $k$ nonsingular $n \times n$ matrices $B_{i}$ and there are given $k$ points $x_{i}$ such that $a<x_{1}<x_{2}<\cdots<x_{k}<b$. Let $a=x_{0}$ and $b=x_{k+1}$. An interface problem is to find a vector $Y(x)$ with $n$ components satisfying a suitable set of boundary conditions, say

$$
Y(a)=Y_{0}
$$

or

$$
A Y(a)+B Y(b)=0
$$

such that

(1.15) on each open subinterval $\left(x_{i-1}, x_{i}\right), Y(x)$ is absolutely continuous; the right and left hand limits $Y\left(x_{i}^{+}\right)$and $Y\left(x_{i}^{-}\right)$of $Y(x)$ at $x=x_{i}$ exist and satisfy

$$
Y\left(x_{i}^{+}\right)=B_{i} Y\left(x_{i}^{-}\right)
$$$$
i=1,2, \cdots k
$$

and

$$
Y^{\prime}=P Y
$$

a.e. on $I$.

In $\S 3$ it is shown how to construct a matrix $R \in R_{n}(I)$ such that solutions of type $R$ of (1.14) or (1.14') and (1.17) also satisfy (1.15) and (1.16). This construction is easily generalized to cover certain cases with infinitely many interfaces $[6$, p. 60]. Since the solutions of interface problems are of type $R$ for some $R \in \Omega_{n}(I)$ it follows from Theorem 2 that interface problems can be reduced to the study of absolutely continuous solutions of (1.7) and suitable boundary conditions.

Turning to the question of equivalent integral equations, for $x \in I$ and $y \in I$, let $W_{n}(I)$ denote the class of $n \times n$ matrices $M(x, y)$ with the following properties:

(1.18) As a function of $x, M(x, y)$ is of bounded variation on $I$ for each $y$.

(1.19) There exists a $\delta$ such that $|\operatorname{det} M(x, y)| \geqq \delta>0$ for each pair $(x, y)$.

(1.20) If $x, y, z$, are any three numbers on $I$ then $M(x, y) M(y, z)$ $=M(x, z)$ and $M(x, x)=E$.

THEOREM 3. $M$ is in class $\varpi_{n}(I)$ if and only if there exists a matrix $V$ of bounded variation on $I$ such that $V^{-1}$ is also of bounded variation on $I$ and 


$$
M(x, y)=V(x) V^{-1}(y) .
$$

Let $\mathfrak{F C}_{n}(I)$ denote the class of harmonic matrices defined by $\mathrm{H} . \mathrm{S}$. Wall [7, p. 160].

LEMma. $M(x, y)$ is harmonic if and only if $M \in W_{n}(I)$ and $M$ is continuous in $x$ for each $y \in I$.

Given the Stieltjes integral equation

$$
Z(x)=C+\int_{x}^{y} d F(s) Z(s) \quad(x, y \in I),
$$

where $Z$ and $C$ are $n \times 1$ matrices and $F$ is an $n \times n$ matrix which is continuous and of bounded variation on $I$. Wall has shown that there exists a unique harmonic matrix $M(x, y)$ satisfying the equation

$$
M(x, y)=E+\int_{x}^{y} d F(s) M(s, y) \quad(x, y \in I),
$$

and that the unique solution of (1.22) is given by $Z(x)=M(x, y) C$.

THEOREM 4. There exists an $R \in R_{n}(I)$ such that the integral equation (1.23) with Stieltjes integral is equivalent to

$$
\psi(x, y)=R(x) R^{-1}(y)+\int_{x}^{y} R(x) R^{-1}(s) P(s) \psi(s, y) d s
$$

where $P=F^{\prime}$ and the integral is in the sense of Lebesgue.

Here equivalence means that if $M(x, y)$ is a solution of (1.23), and if $\psi(x, y)$ is a solution of (1.24) then

$$
M(x, y) \equiv \psi(x, y) .
$$

Since the Stieltjes integral in (1.23) may not exist if $M(x, y)$ is not continuous in $x$, it is necessary that $R(x)$ be continuous if (1.24) is to be equivalent to (1.23). But (1.24) has a solution $\psi(x, y)$ even if $R(x)$ is not continuous and $\psi(x, y)$ is a fundamental matrix of type $R$ for equation (1.5). J. S. MacNerney $[3 ; 4 ; 5]$ and T. H. Hildebrandt [2] have extended the results of Wall permitting more general integrals in (1.23) than the Stieltjes integral. However, so long as $M(x, y)$ is in class $W_{n}(I),(1.24)$ provides an equivalent equation with Lebesgue integration to these generalizations of (1.23).

2. Proof of Theorem 1. If $F$ is of bounded variation on $I$ then:

(a) $F$ is bounded and Lebesgue integrable on $I$,

(b) given $\rho>0$ there exists a constant $c$ such that 


$$
0<\rho \leqq|\operatorname{det}(F-c E)|<\infty \quad \text { for all } x \text { on } I,
$$

(c) $F^{\prime}$ exists almost everywhere on $I$ and is Lebesgue in tegrable. It follows from (b) that $(F-c E)^{-1}$ exists and is of bounded variation on $I$; hence by $(\mathrm{a}),(F-c E)^{-1}$ is bounded and Lebesgue integrable.

Clearly $(F-c E)^{-1} F^{\prime}$ is Lebesgue integrable; therefore, by a well known existence theorem $[1$, p. 67] there exists a unique absolutely continuous fundamental matrix $\Phi(x)$ such that almost everywhere on $I$

$$
\begin{aligned}
& \Phi^{\prime}+\left[(F-c E)^{-1} F^{\prime}\right] \Phi=0, \\
& \Phi(a)=(F(a)-c E)^{-1},
\end{aligned}
$$

Let

$$
G^{-1}=\Phi \quad \text { and } \quad R=(F-c E) G^{-1}
$$

then obviously

$$
F=(F-c E) G^{-1} G+c E=R G+c E .
$$

It is easy to check that $R, G$, and $c E$ have the desired properties. This completes the proof of Theorem 1 .

REMARK 1. If $F=R G+c E$ and $A$ is any nonsingular constant matrix, then clearly $F=R A A^{-1} G+c E$, and the matrix $R A \in \Omega_{n}(I)$ and $A^{-1} G \in \mathcal{G}_{n}(I)$ so the representation of $F$ is not unique. However, if for a given $c$ it is required that $R$ satisfy the initial condition $R(a)$ $=E, a \in I$, then this representation is unique.

REMARK 2. Obviously if $\operatorname{det} F$ is bounded away from zero then $c$ can be taken to be zero.

REMARK 3. It is well known that if $f$ is a function of bounded variation on $I$ then $f$ can be expressed as the sum of a function $h(x)$ which is absolutely continuous on $I$ and a function $s(x)$ which is of bounded variation and has a derivative $s^{\prime}(x)=0$ a.e. on $I$. In the one by one case this fact can be used to give an elementary proof of Theorem 1. If $f$ is of bounded variation on $I$ then there exists a constant $c$ such that $f(x)+c>0$ on $I$. Hence $f(x)+c=\exp [\ln (f(x)+c)]$. Since $\ln (f(x)+c)$ is of bounded variation, it follows that $\ln (f(x)+c)$ $=g(x)+r(x)$ where $g$ is absolutely continuous on $I$ and $r(x)$ is of bounded variation and $r^{\prime}(x)=0$ a.e. on $I$. Hence $f(x)+c$ $=\exp r(x) \exp g(x)$. Clearly, $\exp r(x)$ is in $R_{1}(I)$ and exp $g(x)$ is in $\mathcal{G}_{1}(I)$. This establishes the theorem for the case $n=1$.

3. Interface problems. Let $S_{i}$ be the step function defined by 


$$
S_{i}(x)=\left\{\begin{array}{ll}
E, & a \leqq x<x_{i}, \\
B_{i}, & x_{i} \leqq x \leqq b,
\end{array} \quad(i=1,2, \cdots, k)\right.
$$

and let $R$ be defined by

$$
R(x)=S_{k}(x) S_{k-1}(x) \cdots S_{2}(x) S_{1}(x) .
$$

Clearly for $R$ defined by (3.1) $R \in R_{n}(I)$. It easily follows that a solution of the interface problem $(1.14,1.16,1.17)$ is the solution of type $R$ of $(1.14,1.17)$. By Theorem 2 the interface problem is reduced to finding the absolutely continuous solution of $(1.7,1.8)$ with $R$ as defined above. If $R$ and $\hat{R}$ are considered equivalent if $R=\hat{R}$ except possibly at $x=x_{i}(i=1, \cdots, k)$, then the solution of the interface problem $(1.14,1.16,1.17)$ is unique.

With $R$ defined by (3.1) and $V$ a fundamental matrix of type $R$ of (1.17) the solution of the interface problem $(1.11,1.14,1.16)$ is given by

$$
Y(x)=V(x) V^{-1}(a) Y(a)+\int_{a}^{x} V(x) V^{-1}(s) Q(s) d s
$$

and the solution of the interface problem $\left(1.11,1.14^{\prime}, 1.16\right)$ is given by

$$
Y(x)=\int_{a}^{b} H(x, t) Q(t) d t
$$

where $H(x, t)$ is defined by (1.13).

\section{Equivalent integral systems.}

Proof of Theorem 3. Suppose that $a \in I$ and $M \in W_{n}(I)$. Then by (1.18), $V(x)=M(x, a)$ is of bounded variation on $I$ and (1.19) implies $V^{-1}$ is also of bounded variation on $I$. But (1.20) implies $V^{-1}(x)=M(a, x)$ and $V(x) V^{-1}(y)=M(x, a) M(a, y)=M(x, y)$.

Suppose $V$ and $V^{-1}$ are of bounded variation on $I$. Let $M(x, y)$ $=V(x) V^{-1}(y)$. Clearly $M$ satisfies $(1.18,1.19,1.20)$; hence $M \in W_{n}(I)$.

Let $\mathcal{H C}_{n}(I)$ denote the class of $n \times n$ matrices $M(x, y)$ which satisfy $(1.18,1.20)$ and which are continuous on $I$ in each variable separately. H. S. Wall calls these matrices harmonic.

Proof of Lemma. Let $M(x, y)$ satisfy $(1.18,1.20)$ and note that by (1.20) and properties of determinants

$$
\operatorname{det} M(x, y)=\operatorname{det} M(x, a) \operatorname{det} M(a, y)=\operatorname{det} V(x) \operatorname{det} V^{-1}(y) .
$$

If $M(x, y)$ is continuous in $x$, then $V(x)$ is continuous. By $(1.20) V^{-1}$ exists on $I$ and is therefore continuous. Thus $M(x, y) \in \mathcal{H}_{n}(I)$. Now since $V(x)$ and $V^{-1}(y)$ are continuous and nonsingular det $V(x)$ and 
$\operatorname{det} V^{-1}(y)$ are both different from zero on $I$ and in fact bounded away from zero. Hence (1.19) is satisfied and $\mathfrak{H}_{n}(I) \subset W_{n}(I)$. This completes the proof.

If $M(x, y) \in W_{n}(I)$, then by Theorem 3 there exists a $V$ such that $M(x, y)=V(x) V^{-1}(y)$. Since $V$ is of bounded variation on $I$ by Theorem 1 there exist $R \in \mathcal{R}_{n}(I)$ and $G \in \mathcal{G}_{n}(I)$ such that $V=R G$. Then $V^{-1}=G^{-1} R^{-1}$ and

$$
M(x, y)=R(x) G(x) G^{-1}(y) R^{-1}(y) .
$$

Proof of Theorem 4. If $M(x, y)$ is a solution of $(1.23), M(x, y)$ is harmonic $[7$, Theorem 1, p. 160] and a.e. on $I$

$$
\frac{\partial}{\partial x} M(x, y)=-F^{\prime} M(x, y) \quad(x \in I, y \in I) .
$$

Let $P=F^{\prime}$. Since $M$ is harmonic $M \in W_{n}(I)$ and can be written as in (4.1). Hence for each $y$ on $I M(x, y)$ is a solution of type $R$ on $I$ of the system

$$
\begin{aligned}
Y^{\prime}(x) & =-P(x) Y(x), \\
Y(y) & =E .
\end{aligned}
$$

But by Theorem 2 solving this system is equivalent to finding the absolutely continuous solution $W$ of

$$
\begin{aligned}
W^{\prime}(x) & =-R^{-1}(x) P(x) R(x) W(x), \\
W(y) & =R^{-1}(y)
\end{aligned}
$$

and multiplying on the left by $R(x)$. It is well known that an integral equation equivalent to $(4.4,4.5)$ is

$$
W(x, y)=R^{-1}(y)+\int_{y}^{x}-R^{-1}(s) P(s) R(s) W(s, y) d s .
$$

Let $\psi(x, y)=R(x) W(x, y)$. Then $\psi(x, y)$ satisfies (1.24); that is,

$$
\psi(x, y)=R(x) R^{-1}(y)+\int_{x}^{y} R(x) R^{-1}(s) P(s) \psi(s, y) d s .
$$

In fact $M(x, y) \equiv \psi(x, y),(x \in I, y \in I)$.

\section{REFERENCES}

1. E. A. Coddington, and N. Levinson, Theory of ordinary differential equations, McGraw-Hill, New York, 1955.

2. T. H. Hildebrandt, On systems of linear differentio-Stieltjes integral equations, Illinois J. Math. 3 (1959), 352-373. 
3. J. S. MacNerney, Stieltjes integrals in linear spaces, Ann. of Math. (2) 61 (1955), 354-367.

4. - Continuous products in linear spaces, J. Elisha Mitchell Sci. Soc. 71 (1955), 185-200.

5. - Determinants of harmonic matrices, Proc. Amer. Math. Soc. 7 (1956), 1044-1046.

6. F. W. Stallard, Differential systems with interface conditions, Oak Ridge National Laboratory Publication no. 1876 (Physics).

7. H. S. Wall, Concerning harmonic matrices, Arch. Math. 5 (1954), 160-167.

Georgia Institute of Technology

\section{ASYMPTOTIC BEHAVIOR OF THE SOLUTIONS OF NONLINEAR DIFFERENTIAL EQUATIONS}

T. F. BRIDGLAND, JR.

I. Of considerable significance for the stability analysis of signal transmission systems is the relation between the boundedness and asymptotic behavior of the solutions of the linear differential equation

$$
\frac{d y}{d t}=A(t) y+p(t)
$$

and of the solutions of the nonlinear equation

$$
\frac{d z}{d t}=A(t) z+\phi(z ; t)
$$

Several results on this relationship have been obtained by Perron [1], Bellman [2], Coddington and Levinson [3] and others. The results of the present note-which we state after suitable restriction of (1) and (2)-are further theorems on this relationship.

In (1) and (2) we suppose that the $n \times n$ matrix $A(t)$ has elements which are real-valued, continuous and bounded for $t \geqq 0$ while $p(t)$, $\phi(z ; t)$ are $n$-vectors with the former having elements which are realvalued and continuous for $t \geqq 0$ and the latter having elements which are real-valued and continuous for all $t \geqq 0$ and all $z \in V$, where $V$ is some neighborhood of $z=0$ in the space of $n$-tuples of real numbers.

Received by the editors November 2, 1960 and, in revised form, April 24, 1961. 